\title{
Practical Binary Adaptive Block Coder
}

\author{
Yuriy A. Reznik \\ QUALCOMM Incorporated \\ 5775 Morehouse Drive, San Diego, CA 92121 \\ E-mail: yreznik@ieee.org
}

\begin{abstract}
This paper describes design of a low-complexity algorithm for adaptive encoding/decoding of binary sequences produced by memoryless sources. The algorithm implements universal block codes constructed for a set of contexts identified by the numbers of non-zero bits in previous bits in a sequence.

We derive a precise formula for asymptotic redundancy of such codes, which refines previous well-known estimate by Krichevsky and Trofimov [24, and provide experimental verification of this result.

In our experimental study we also compare our implementation with existing binary adaptive encoders, such as JBIG's Q-coder [4], and MPEG AVC (ITUT H.264)'s CABAC [43] algorithms.
\end{abstract}

\section{Introduction}

One of the most basic tasks in the design of today's data compression algorithms is the one of converting input sequences of bits with some unknown distribution into a decodable bitstream. This happens, for example, in the design of image or video codecs, scalable (bit-slice based) encoding of spectrum in audio codecs, etc. In most such cases, the bits to be encoded are taken from values produced by various signal processing tools (transforms, prediction filters, etc), which means that they are already well de-correlated, and that assumption of memorylessness of such a source is justified.

Most commonly, the problem of encoding of such sequences of bits is solved by using fast (typically multiplication-free) approximations of binary adaptive arithmetic codes. Two well known examples of such algorithms are IBM's Q-coder [41] adopted in JBIG image coding standard [42], and CABAC encoder [43] used in MPEG AVC/ITU-T H.264 standards for video compession [44].

In this paper we describe an alternative implementation of adaptive encoder using an array of Huffman codes designed for several estimated densities, indexed by the numbers of non-zero bits in previous blocks (contexts) in a sequence.

We study both efficiency and implementation aspects of such a scheme and show that by using even relatively short blocks $(8 \ldots 16$ bits, and using correspondingly $1.5 . .5 K$ bytes of memory) it can achieve compression performance comparable or superior to one of the above quoted algorithms.

This paper is organized as follows. In Section 2 we provide background information about our coding problem. In Section 3 we quote known results about efficiency 
of such codes and offer a more precise result. In Sections 4 and 5 we describe design of our system, and in Section 6 we provide experimental results. Appendix A contains proofs of out Theorem 1, and Appendix B contains complete code of the program we've designed.

\section{Background Information}

Consider a memoryless source producing symbols from a binary alphabet $\{0,1\}$ with probabilities $p$, and $q=1-p$ correspondingly. If $w$ is a word of length $n$ produced by this source, then its probability:

$$
\operatorname{Pr}(w)=p^{k} q^{n-k},
$$

where $k$ denotes the number of 1's in this word (sometimes $k$ is also referred to as weight of $w)$.

A block code $\phi$ is an injective mapping between words $w$ of length $|w|=n$ and binary sequences (or codewords) $\phi(w)$ :

$$
\phi:\{0,1\}^{n} \rightarrow\{0,1\}^{*},
$$

where the codewords $\phi(w)$ represent a uniquely decodable set [7].

Typically, when the source (i.e. its probability $p$ ) is known, such a code is designed to minimize its average length, or (in relative terms) its average redundancy:

$$
R_{\phi}(n, p)=\frac{1}{n} \sum_{|w|=n} \operatorname{Pr}(w)|\phi(w)|-H(p) .
$$

As customary by $H(p)=-p \log p-q \log q$ we denote the entropy of the source [7].

Classical examples of codes and algorithms suggested for solving this problem include Huffman [18, Shannon [34], Shannon-Fano [12, Gilbert-Moore [17] codes and their variants [1]. Performance of such codes is well studied, see, e.g. [16], [25], [35, [36], 30. Analysis of their complexity can be found in [38], 39]. Many useful practical implementation techniques for such codes have also been reported, see, e.g. [1], [27], [4].

When the source is not known, the best option available is to design a universal code $\phi^{*}$ that minimize the worst case redundancy for a class of sources [13, 8, 25]:

$$
R_{\phi^{*}}(n)=\inf _{\phi} \sup _{p} R_{\phi}(n, p) .
$$

An example of such a code can be constructed using the following estimates of words' probabilities: 1 :

$$
P_{K T}(w)=\frac{\Gamma(k+1 / 2) \Gamma(n-k+1 / 2)}{\pi \Gamma(n+1)},
$$

where $\Gamma(x)$ is a $\Gamma$-function, $k$ is the weight of word $w$, and $n$ is its length.

Finally, we might be in a situation when exact value of parameter of the source is not known, but we can access a sequence of symbols $u$ produced by this source in

\footnotetext{
${ }^{1}$ This formula is due to Krichevsky and Trofimov [24, and it assures uniform (in $p$ ) convergence to true probabilities with $n \rightarrow \infty$. See [26] and 40] for discussions on its background and optimality.
} 
the past. We will call such a sequence a sample, and will assume that it is $|u|=t$ bits long. The task here is to design a set of codes (indexed by different values of this sample) $\phi_{u}^{*}$, such that their resulting worst case average redundancy is minimal:

$$
R_{\phi_{u}^{*}}(n, t)=\inf _{\left\{\phi_{u}\right\}} \sup _{p} \sum_{|u|=t} \operatorname{Pr}(u) R_{\phi_{u}}(n, p) .
$$

Such codes are called sample-based or adaptive universal block codes [22, 23, 24].

In this paper we will study a particular implementation of adaptive block codes utilizing the following estimates of probabilities of words $w$ given a sample $u$ :

$$
P_{K T}(w \mid u)=\frac{P_{K T}(u w)}{P_{K T}(u)}=\frac{\Gamma(k+s+1 / 2) \Gamma(n+t-k-s+1 / 2)}{\Gamma(s+1 / 2) \Gamma(t-s+1 / 2)} \frac{\Gamma(t+1)}{\Gamma(n+1)},
$$

where $s$ is the weight of a sample $u$, and $t$ is its length.

\section{Performance of Adaptive Block Codes}

The idea and original analysis of sample-based codes utilizing estimator (6) belong to R. E. Krichevsky [23]. In particular, he has shown (cf. [24, Theorem 1], [25, Theorem 3.4.1]), that the average redundancy rate of an adaptive block code is asymptotically

$$
R_{\phi_{u}^{*}}(n, t) \sim \frac{1}{2 n} \log \frac{n+t}{t},
$$

where $n$ is a block size, and $t$ is the size of samples.

From (77) it is clear, that by using samples of length $t=O(n)$ it is possible to lower redundancy rate of such codes to $O\left(\frac{1}{n}\right)$, which matches the order of redundancy rate of block codes for known sources [7, 25, 36.

However, in order to be able to understand full potential of such codes it is desirable to know more exact expression for their redundancy, including terms affected by the choice of actual code-construction algorithm (such as Huffman, Shannon, etc). The theorem below 2 offers such a refinement.

Theorem 1 (Reznik \& Szpankowsky 2003) The average redundancy rate of an adaptive block code $\phi_{u}^{*}$ has the following asymptotic behavior $(n, t \rightarrow \infty)$ :

$$
\begin{aligned}
& R_{\phi_{u}^{*}}(n, t, p)=\sum_{|u|=t} \operatorname{Pr}(u) R_{\phi_{u}^{*}}(n, p) \\
&=\frac{1}{n}\left\{\frac{1}{2} \log \frac{t+n}{t}+\Delta_{\phi_{u}^{*}}(n, t, p)+\frac{1-4 p q}{24 p q} \frac{n}{t(t+n)}-\frac{1-3 p q}{24 p^{2} q^{2}} \frac{(n+2 t) n}{t^{2}(t+n)^{2}}\right. \\
&\left.\quad+O\left(\frac{1}{t^{3}}+\frac{1}{n^{3}}\right)\right\},
\end{aligned}
$$

where $n$ is a block size, and $t$ is a sample size, $p, q=1-p$ are probabilities of symbols of the input source, and where

$$
\Delta_{\phi_{u}^{*}}(n, t, p)=\sum_{|u|=t} \sum_{|w|=n} \operatorname{Pr}(u) \operatorname{Pr}(w)\left[\left|\phi_{u}^{*}(w)\right|+\log P_{K T}(w \mid u)\right]
$$

is the average redundancy of code $\phi_{u}^{*}$ with respect to estimated distribution (6)).

\footnotetext{
${ }^{2}$ This is a simple generalization of our previous result for adaptive Shannon codes [29].
} 


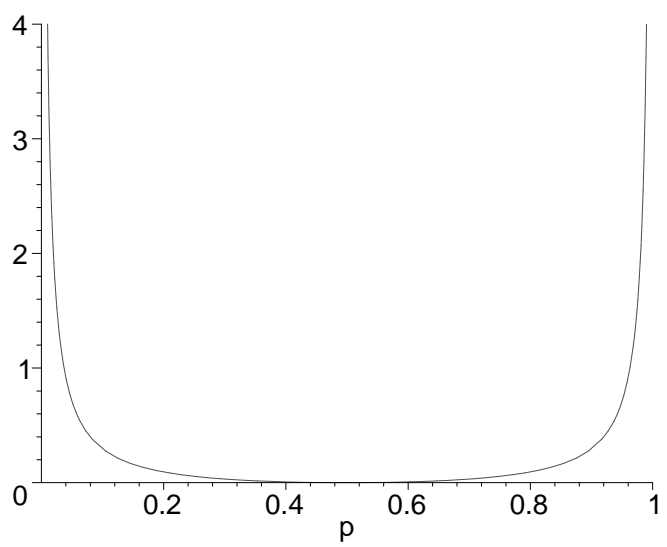

Figure 1: Behavior of a factor $\frac{1-4 p q}{24 p q}$ in redundancy expression (8).

The exact behavior of $\Delta_{\phi_{u}^{*}}(n, t, p)$ is algorithm-specific, but for a large class of minimum-redundancy techniques, which includes conventional Huffman and Shannon codes, we can say that this term is bounded in magnitude

$$
|\Delta(n, t, S)| \leqslant 1
$$

and that it exhibits oscillating behavior, which may or may not be convergent to some constant depending on the value of the parameter $p$ (cf. [36, [9], 29]).

We also notice, that for short $t$ and $n$ the redundancy of such codes becomes affected by the next following term:

$$
\frac{1-4 p q}{24 p q} \frac{n}{t(t+n)}
$$

which is a function of the parameter of the source $p$. We plot leading factor of this term in Figure 1, and conclude that for short blocks/samples performance of such codes becomes sensitive to the asymmetry of the source.

Proof of this theorem can be found in Appendix A, and the rest of paper is devoted to study of efficient algorithms for implementing such codes.

\section{Efficient Implementation of Block Codes}

We first notice, that in a memoryless model the probability of a word $w$ (or its estimate, cf. (1), (4), (6) ) depends only on its weight $k$, but not an actual pattern of its bits. Hence, considering a set of all possible $n$-bit words, we can split it in $n+1$ groups:

$$
\{0,1\}^{n}=W_{n, 0} \cup W_{n, 1} \cup \ldots \cup W_{n, k} \cup \ldots \cup W_{n, n},
$$

containing words of the same weight $(k=0, \ldots, n)$, and the same probability. As obvious, the sizes of such groups are $\left|W_{n, k}\right|=\left(\begin{array}{l}n \\ k\end{array}\right)$. For further convenience, we will assume that each group $W_{n, k}$ stores words in a lexicographic order. By $I_{n, k}(w)$ we will denote the index (position) of a word $w$ in a group $W_{n, k}$.

To describe the structure of our proposed mapping between words in groups $W_{n, k}$ and their codewords, we will use an example code shown in Table 1. This 
Table 1: Example of a code constructed for 4-bit blocks with Bernoulli probabilities: $p^{k} q^{n-k}, p=0.9$.

\begin{tabular}{|c|c|c|c|c|l|c|}
\hline \hline Block $w$ & $k$ & $I_{n, k}(w)$ & $\operatorname{Pr}(w)$ & Length & Code $\phi(w)$ & Sub-group \\
\hline 0000 & 0 & 0 & 0.6561 & 1 & 1 & 0 \\
0001 & 1 & 0 & 0.0729 & 3 & 001 & 1 \\
0010 & 1 & 1 & 0.0729 & 3 & 010 & 1 \\
0011 & 2 & 0 & 0.0081 & 6 & 000011 & 3 \\
0100 & 1 & 2 & 0.0729 & 3 & 011 & 1 \\
0101 & 2 & 1 & 0.0081 & 7 & 0000001 & 4 \\
0110 & 2 & 2 & 0.0081 & 7 & 0000010 & 4 \\
0111 & 3 & 0 & 0.0009 & 9 & 000000001 & 5 \\
1000 & 1 & 3 & 0.0729 & 4 & 0001 & 2 \\
1001 & 2 & 3 & 0.0081 & 7 & 0000011 & 4 \\
1010 & 2 & 4 & 0.0081 & 7 & 0000100 & 4 \\
1011 & 3 & 1 & 0.0009 & 9 & 000000010 & 5 \\
1100 & 2 & 5 & 0.0081 & 7 & 0000101 & 4 \\
1101 & 3 & 2 & 0.0009 & 9 & 000000011 & 5 \\
1110 & 3 & 3 & 0.0009 & 10 & 0000000001 & 6 \\
1111 & 4 & 0 & 0.0001 & 10 & 0000000000 & 7 \\
\hline \hline
\end{tabular}

code was constructed using a modification of Huffman's algorithm 18, in which additional steps were taken to ensure that codewords located at the same level have same lexicographic order as input blocks that they represent. It is well-known that such a reordering is possible without any loss of compression efficiency, and examples of prior algorithms that have been using this idea include Huffman-Shannon-Fano codes [5, canonic codes of Moffat and Turpin [27], 4], etc.

In Figure 2 we depict the structure of this code. As expected, each group $W_{n, k}$ consists of at most two sub-groups containing codewords of the same length: 3

$$
W_{n, k}=W_{n, k, \ell} \cup W_{n, k, \ell+1},
$$

where $\ell$ is the shortest code length that can be assigned to blocks from $W_{n, k}$. Moreover, since words within $W_{n, k}$ group follow lexicographic order, then the split between $W_{n, k, \ell}$ and $W_{n, k, \ell+1}$ is simply:

$$
\begin{aligned}
W_{n, k, \ell} & =\left\{w \in W_{n, k}: I_{n, k}(w)<n_{k}\right\}, \\
W_{n, k, \ell+1} & =\left\{w \in W_{n, k}: I_{n, k}(w) \geqslant n_{k}\right\}
\end{aligned}
$$

where $n_{k}$ denotes the size of a subgroup with shorter codewords.

We will call lexicographically smallest codewords in each subgroup base codewords:

$$
\begin{aligned}
B_{n, k, \ell} & =\phi\left(w_{0}\right), \\
B_{n, k, \ell+1} & =\phi\left(w_{n_{k}}\right),
\end{aligned}
$$

\footnotetext{
${ }^{3}$ This follows from the fact that all words in $W_{n, k}$ have the same probability, and so-called sibling property of Huffman codes (cf. [16, 35], 36]). This observation also holds true for Generalized Shannon codes 10 and possibly some other algorithms.
} 


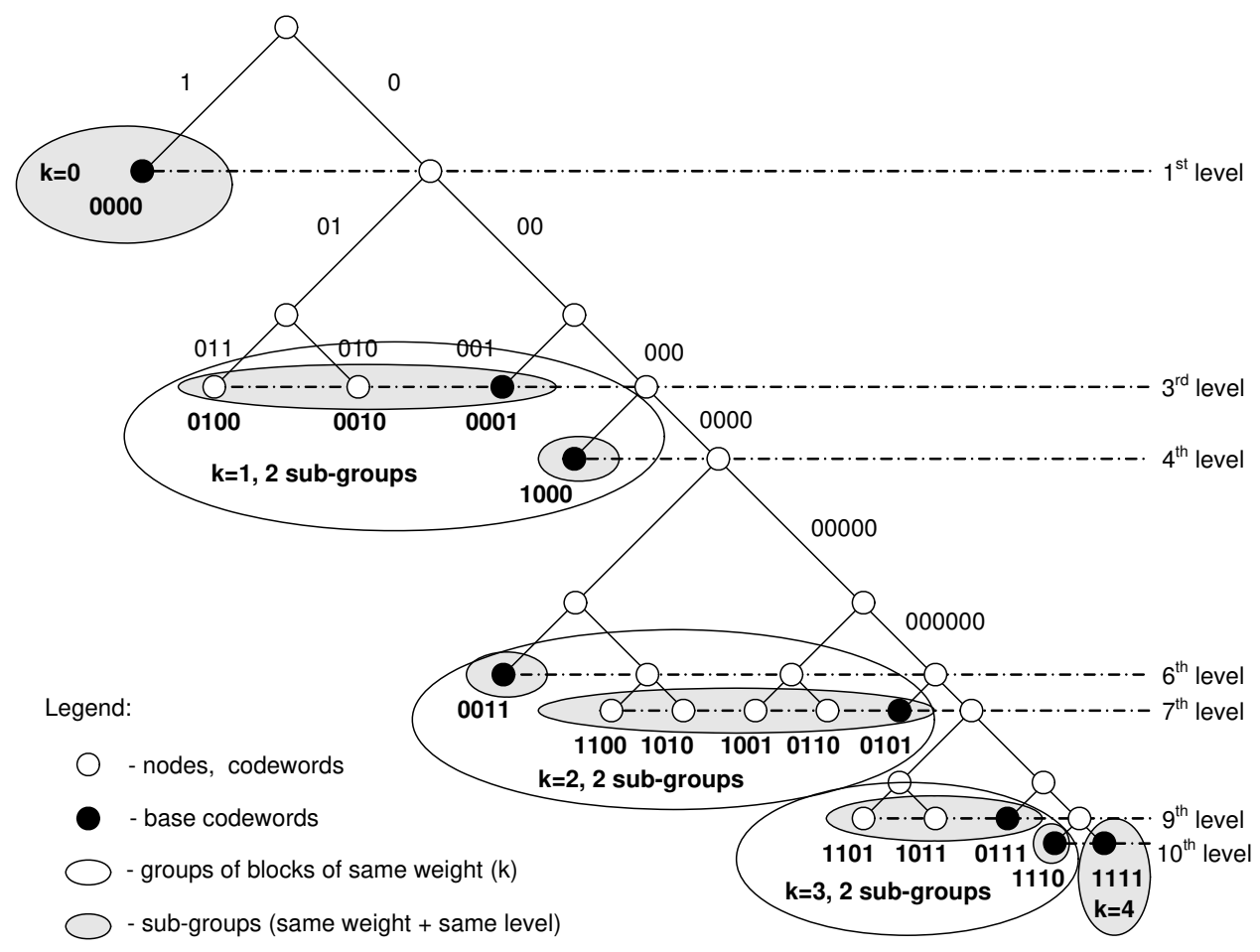

Figure 2: Structure of an example block code.

where $w_{i}$ : is $i$-th block in $W_{n, k}$, and note that the remaining codewords in both subgroups can be computed as follows:

$$
\phi\left(w_{i}\right)=\left[\begin{array}{ll}
B_{n, k, \ell}+i, & \text { if } i<n_{k}, \\
B_{n, k, \ell+1}+i-n_{k}, & \text { if } i \geqslant n_{k}
\end{array}\right.
$$

We point out that such base codewords are only defined for non-empty subgroups, and that the number of such subgroups $S$ in a tree constructed for $n$-bit blocks is within:

$$
n+1 \leqslant S \leqslant 2 n .
$$

We also notice that multiple subgroups can reside on the same level 4 (see e.g. level 10 in tree in Figure 2), and the number of such collocated sub-groups cannot be greater than $n+1$.

\subsection{Proposed Algorithm for Block Encoding/Decoding}

Based on the discussion above we can now define a simple algorithm for direct computation of block codes.

We assume that parameters $n_{k}(0 \leqslant k \leqslant n)$ are available, and that for each non-empty sub-group we can obtain its level $\ell$ and its base codeword $B_{n, k, \ell}$. Then the process of encoding a block $w$ is essentially a set of the following steps:

\footnotetext{
${ }^{4}$ This is one of the most obvious differences between our algorithm and Connell [5], or Moffat and Turpin 27] algorithms, which assign unique base codewords for each level, but then they need an $O\left(n 2^{n}\right)$-large reordering table to work with such codes. Here, the entire structure is $O\left(n^{2}\right)$ bits large. Also, unlike 38, 39] our algorithm does not assume any particular order of probabilities based on weight $k$. This way we can implement codes for universal densities (4), and (6).
} 


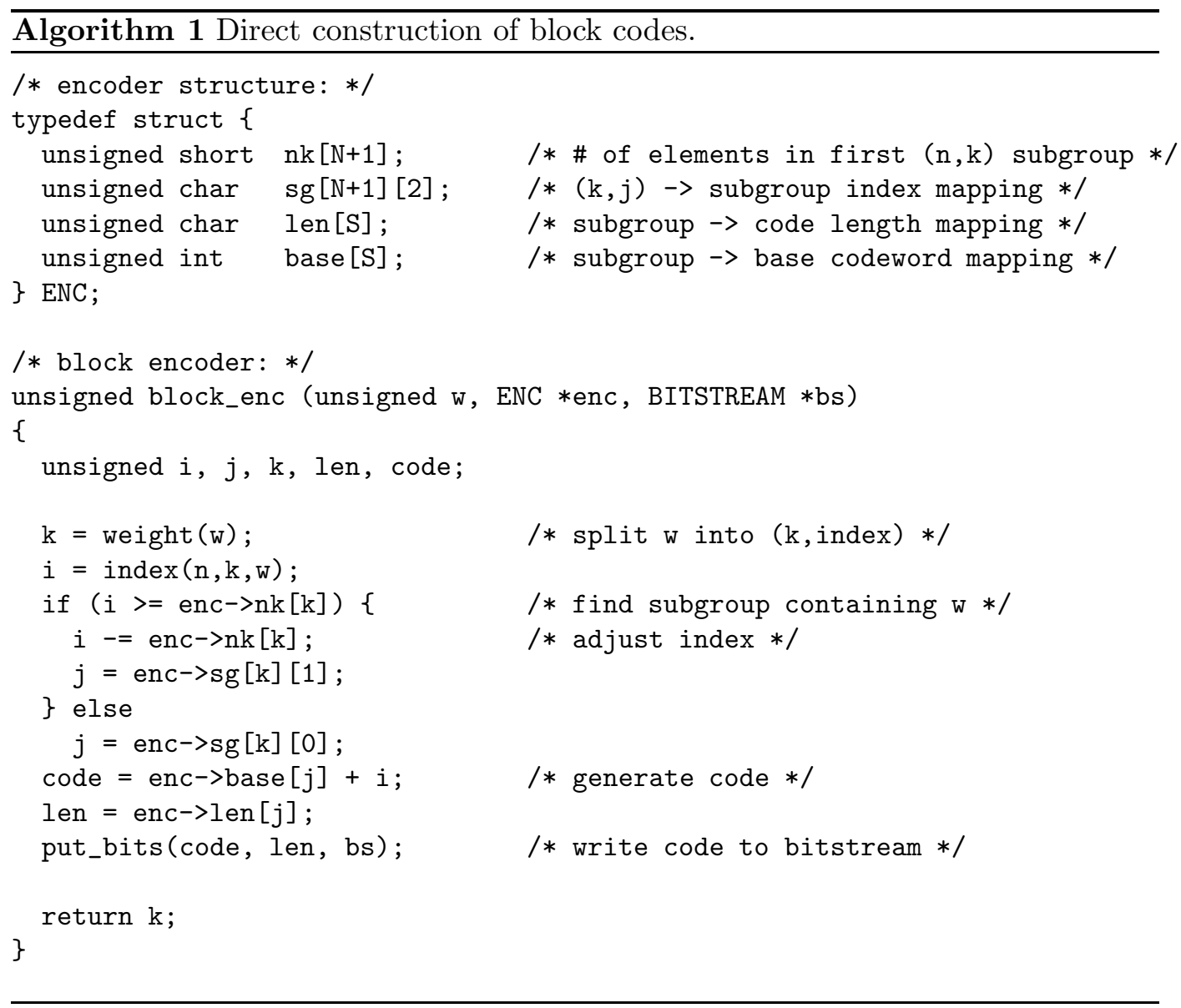

- using $w$ obtain its weight $k$, and index $I_{n, k}(w)$

- if $I_{n, k}(w)<n_{k}$ use first subgroup $W_{n, k, \ell}$ otherwise pick $W_{n, k, \ell+1}$

- retrieve base codeword and compute the code according to (16).

A complete C-language code of such a procedure is presented as Algorithm 1 above.

It can be seen that memory-wise this algorithm needs only $S$ base codewords $(O(n)$-bit long 5$), n+1$ values $n_{k}(O(n)$-bit long), $S$ code lengths $(O(\log n)$-bit long), and $2(n+1)$ subgroup indices $(O(\log n)$-bit long). Given the fact that $S=O(n)$, the entire structure needs $O\left(n^{2}\right)$ bits.

In a particular implementation shown in Algorithm 1, and assuming, e.g. that $n=20$ and $S=32$, the size of this structure becomes 244 bytes - far less than $2^{20}$ words needed to present this code in a form of a direct table.

We note that for reasonably short blocks (e.g. $n \leqslant 12 \ldots 16$ ) computation of their weights and indices (functions weight(.) and index (.,.) in Algorithm 1), can be a matter of a single lookup, in which case, the entire encoding algorithm needs at most 1 comparison, 2 additions, and 4 lookups.

\footnotetext{
${ }^{5}$ We note that additional memory reduction is possible by storing incremental values of base codewords - this is discussed in a companion paper [32.
} 
For larger blocks, one can use the following well-known combinatorial formula (cf. [28], [2], [33], 6], [38], 39]):

$$
I_{n, k}(w)=\sum_{j=1}^{n} w_{j}\left(\begin{array}{c}
n-j \\
\sum_{k=j}^{n} w_{k}
\end{array}\right),
$$

where $w_{j}$ represent individual bits of the word $w$, and it is assumed that $\left(\begin{array}{l}n \\ k\end{array}\right)=0$ for all $k>n$. In order to implement it, one could either pre-compute all binomial coefficients up to level $n$ in Pascal's triangle, or compute them dynamically, using the following simple identities:

$$
\left(\begin{array}{l}
n-k \\
k-1
\end{array}\right)=\frac{k}{n}\left(\begin{array}{l}
n \\
k
\end{array}\right), \text { and }\left(\begin{array}{c}
n-k \\
k
\end{array}\right)=\frac{n-k}{n}\left(\begin{array}{l}
n \\
k
\end{array}\right) .
$$

The implementation based on pre-computed coefficients requires $\frac{n(n+1)}{2}=O\left(n^{2}\right)$ words $\left(O\left(n^{3}\right)\right.$ bits) of memory, and $O(n)$ additions. Dynamic computation of coefficients will require $O(n)$ additions, multiplications and divisions, but the entire process needs only few registers. Additional discussion on complexity of index computation can be found in [39.

We now turn our attention to the design of a decoder. Here, we will also need parameters $n_{k}$, base codewords, and their lengths. For further convenience (as it was suggested by Moffat and Turpin [27]) we will use left-justified versions of base values:

$$
B_{n, k, \ell}^{l j}=B_{n, k, \ell} 2^{T-\ell},
$$

where $T$ is the length of a machine word $(T>\max \ell)$. We will store such leftjustified values in a lexicographically decreasing order. Then, the decoding process can be described as follows:

- find first (top-most) subgroup with $B_{n, k, \ell}^{l j}$ being less than last $T$ bits in bitstream,

- decode index of a block $I_{n, k}(w)$ (based on (16)), and

- produce reconstructed block using its weight $k$ and index.

A complete C-language code of such a procedure is presented as Algorithm 2.

We note that (besides using left-justified base words) this algorithm has almost identical data structure. The only new elements here are weights $k$ and subgroup level indicators $j$ ( $j=0$ if subgroup contains shorter codewords, and $j=1$ otherwise). Memory-wise it has very similar characteristics.

The main decoding process requires between 1 and $S$ comparisons and lookups to find a subgroup, 1 or 2 additions, 1 shift, 1 extra comparison, and 3 extra lookups.

As in Moffat-Turpin algorithm [27] the number of steps needed for finding a subgroup can be further reduced by placing base codewords in a binary search tree or using an extra lookup table, but in both cases we need to use extra memory to accomplish this.

We note, that at the end of the decoding process we also need to convert word's weight $k$ and index $I_{n, k}(w)$ into its actual value (function word () in Algorithm 2). If blocks are reasonably short, this can be accomplished by a simple lookup. Otherwise, we can synthesize the word by using the enumeration formulae (18). Complexitywise this process is similar to index computation in the encoder. 


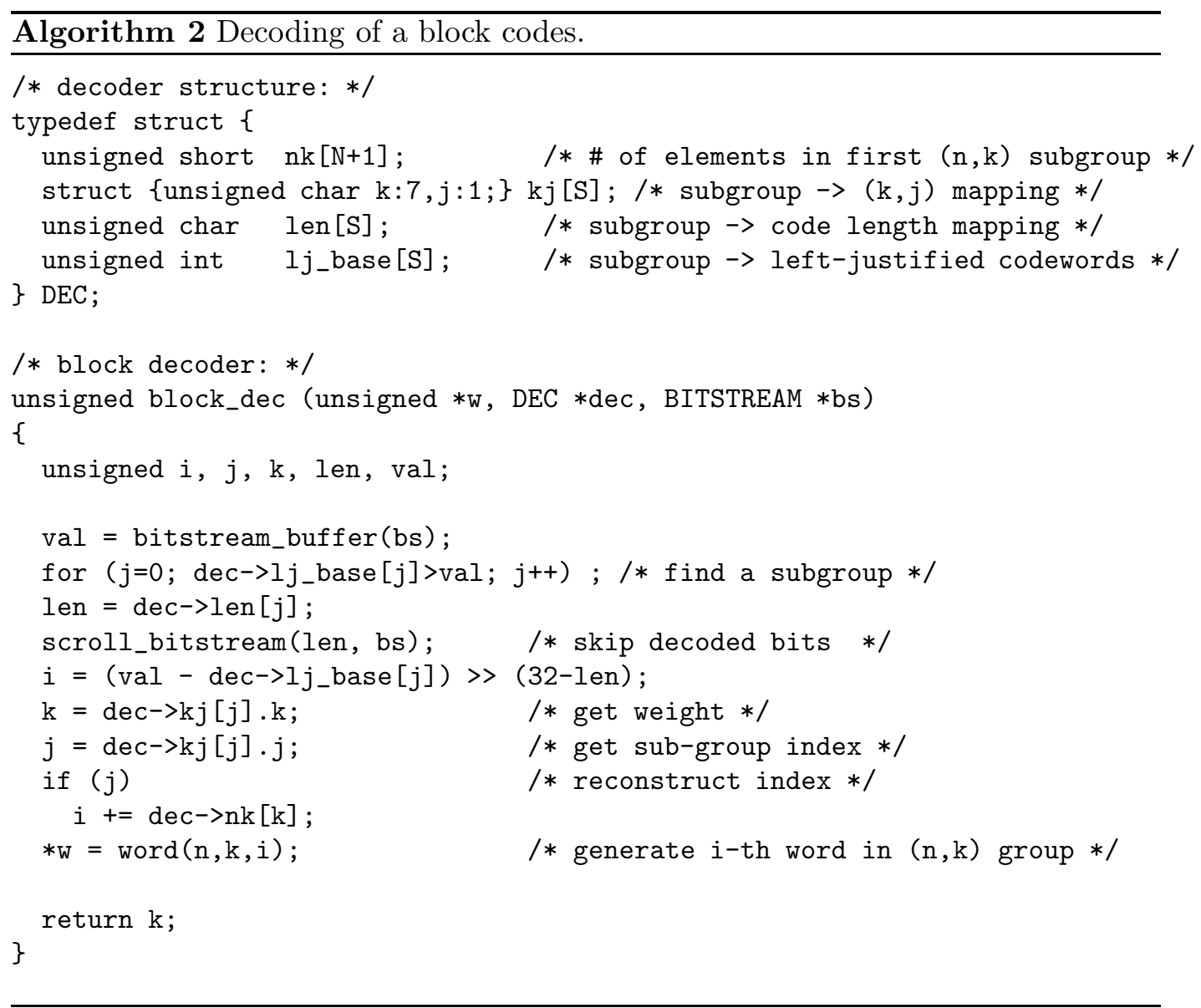

\section{Design of an Adaptive Block Coder}

Using above described algorithms we can now define a system for adaptive encoding/decoding of blocks of data.

In this system, we assume that input blocks can be encoded under the following conditions:

1. there is no context - i.e. we implement universal code,

2. the context is given by one previously seen block - i.e. $t=n$,

3. the context is given by two previously seen blocks - i.e. $t=2 n$.

We note, that instead of using actual blocks as contexts it is sufficient (due to memoryless nature of the source) to use their weights.

This means, that for $t$-bit samples, we will need to have an array of $t+1$ code structures indexed by their weights $s$. To further save space, we can use symmetry of KT-distributions (6) with respect to $s$ and $k$ : replace $s=t-s$ and flip bits (i.e. force $k=n-k)$ every time when $s>t / 2$. This way we will only need to define $t / 2+1$ tables.

Hence, the overall amount of memory needed by our adaptive code becomes $1+n / 2+1+n+1=1.5 n+3$ tables. Specific memory estimates for block sizes $n=8 \ldots 20$, are shown in Table 2 . 
Table 2: Memory usage estimates [in bytes] for different block sizes

\begin{tabular}{|c|c|c|c|c|}
\hline \hline$n$ & $\max t$ & $\max S$ & Size of a single table & Tables for all contexts \\
\hline 8 & 16 & 14 & 102 & 1530 \\
12 & 24 & 19 & 140 & 2940 \\
16 & 32 & 25 & 184 & 4968 \\
20 & 40 & 29 & 216 & 7128 \\
\hline \hline
\end{tabular}

In out test implementation we've generated all these tables using KT-estimated densities (4) and (6), and using modified Huffman code- construction algorithm, as described in Section 3.

In Appendix B we provide a complete code of a program implementing such a system.

\section{Experimental Study of Performance of our Algorithm}

In this section we provide experimental results of evaluation of performance of our adaptive code with block size $n=16$, and compare it with the following well known algorithms:

- IBM's Q-coder algorithm [41 adopted in JBIG standard for image compression [42] (we've used implementation from JBIG's jbigkit);

- CABAC binary arithmetic encoder [43] from MPEG AVC/ITU-T H.264 standard for video coding [44.

In order to conduct our tests we've used computed-generated sequences of bits simulating output from a binary Bernoulli source with probability $p$. Lengths of such sequences ranged from 16 to 1024, and for each particular length we have generated $Q=1000000$ samples of such sequences.

Relative redundancy rates were computed as:

$$
\text { Rate }=\frac{\text { (sum of lengths of all codes produced for Q sequences) } / Q-H(p)}{H(p)}
$$

For our adaptive code we've used the following structure of contexts:

- first 16-bit block is encoded without context (universal code),

- second block is encoded using first one as its context (code with $t=16$ ),

- third and all subsequent blocks are encoded using two previous blocks in a sequence as contexts (sample-based code with $t=32$ ).

The results of our experimental study are shown in Figures 3 and 4 . It can be seen that our code has a much faster rate of convergence than that of Q-coder or CABAC algorithms. It clearly outperforms them for short sequences, and becomes comparable to the best of these two when the total length of encoded bits approaches 1024 . 

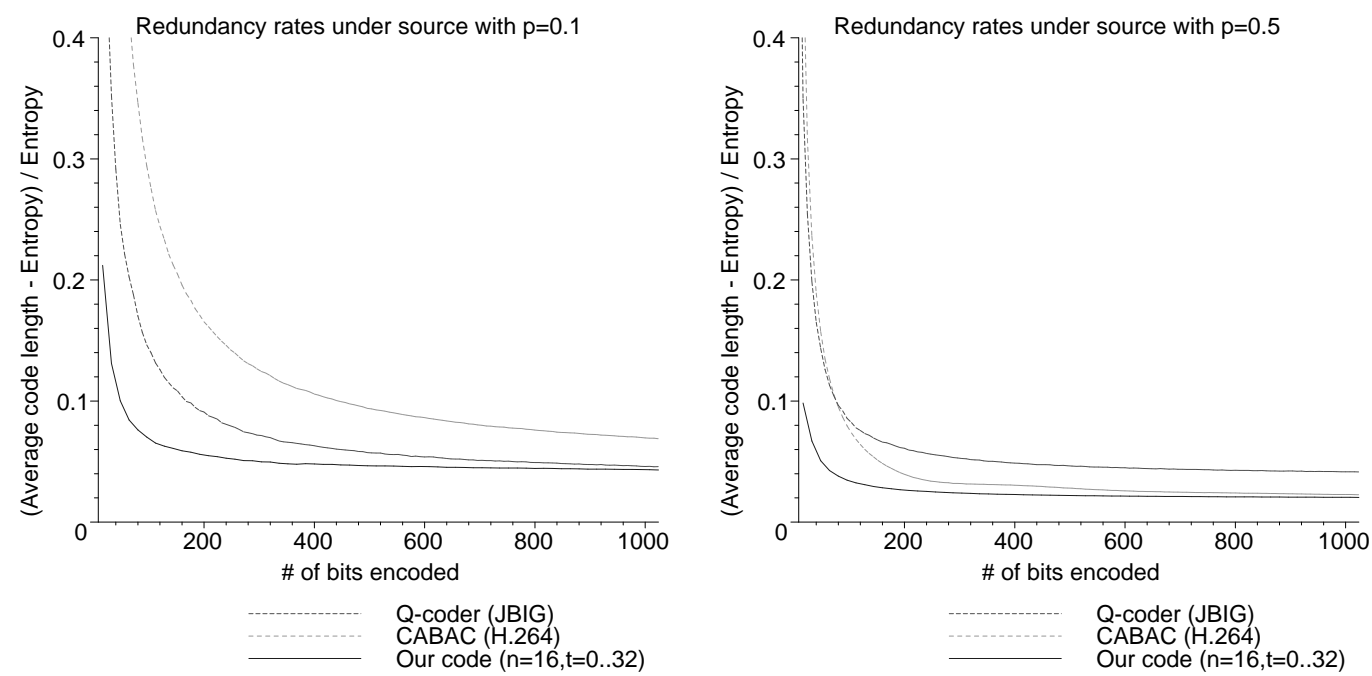

Figure 3: Comparison of redundancy rates under memoryless sources with $p=0.1$ (left) and $p=0.5$ (unbiased case, right).

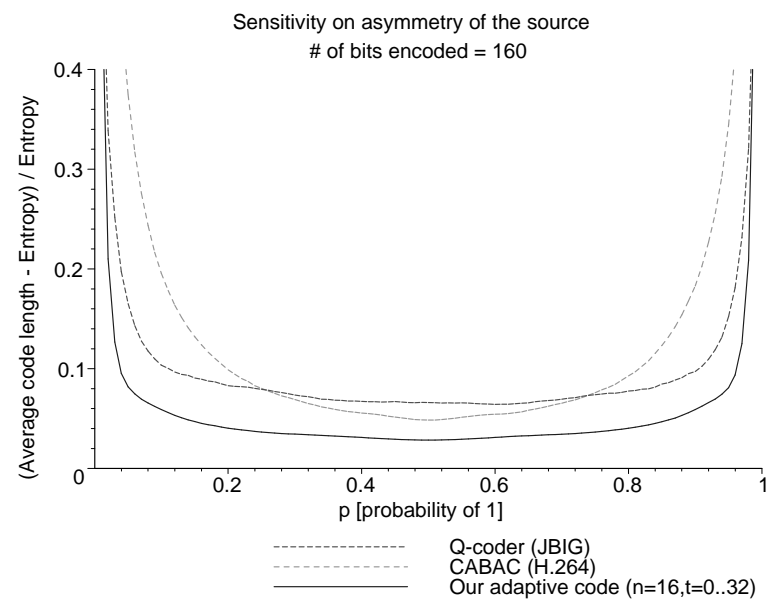

Figure 4: Sensitivity of redundancy to asymmetry of the source.

In Figure 4 we also show analysis of sensitivity of redundancy rates of these codes to asymmetry of the source. Here, after 160 encoded bits (or 10 16-bit blocks) our algorithm delivers much lower redundancy compared to others. Its behavior is consistent with one that was predicted by our Theorem 1 .

\section{References}

[1] J. Abrahams, Code and parse trees for lossless source encoding, in Proc. Compression and Complexity of SEQUENCE'97 (Positano: IEEE Press, 1998) 145-171.

[2] V. F. Babkin, A method of universal coding with non-exponent labour consumption, Probl. Inform. Trans., 1 (4) (1971) 13-21 (in Russian)

[3] A. A. Borovkov, Probability Theory (Gordon \& Breach, 1998) 
[4] A. Brodnik and S. Carlsson, Sublinear Decoding of Huffman Codes Almost in Place, DIMACS Workshop on Codes and Trees: Algorithmic and Information Theoretic Approaches, (October 1998, Rutgers University, DIMACS Center, NJ).

[5] J. B. Connell, A Huffman-Shannon-Fano Code, Proc. IEEE (July 1973) 1046-1047.

[6] T. M. Cover, Enumerative Sources Encoding, IEEE Trans. Inform. Theory, 19 (1) (1973) 73-77.

[7] T. M. Cover and J. M. Thomas, Elements of Information Theory, (John Wiley \& Sons, New York, 1991).

[8] L. D. Davisson, Universal Noiseless Coding, IEEE Trans. Inform. Theory, 19 (6) (1973) 783-795.

[9] M. Drmota, H-K. Hwang, and W. Szpankowski, Precise Average Redundancy of an Idealized Arithmetic Coding, Proc. Data Compression Conference (2002) 222-231.

[10] M. Drmota and W. Szpankowski, Generalized Shannon Code Minimizes the Maximal Redundancy, in Proc. LATIN'02 (Cancun, Mexico, 2002) 306-318.

[11] M. Drmota and R. Tichy, Sequences, Discrepancies, and Applications (Springer-Verlag, Berlin, 1997).

[12] R M. Fano, The transmission of information. Res. Lab. Electronics, Massachusetrs Inst. of Technology, Cambridge, Mass. Tech. Rep. 65 (1949).

[13] B. M. Fitingof, Optimal Coding in the Case of Unknown and Changing Message Statistics, Probl. Inform. Transm., 2, (2) (1965) 3-11 (in Russian) 1-7 (English Transl.)

[14] P. Flajolet, Singularity analysis and asymptotics of Bernoulli sums, Theoretical Computer Science, 215 (1999) 371-381.

[15] P. Flajolet and A. Odlyzko, Singularity analysis of generating functions, SIAM J. Discrete Math., 3 (2) (1990) 216-240.

[16] R. Gallager, Variations on the theme by Huffman, IEEE Trans. Inform. Theory, IT-24 (Nov. 1978) 668-674.

[17] E. N. Gilbert and E. F. Moore, Variable-Length Binary Encodings, Bell Syst. Tech. J., 7 (1959) 932-967.

[18] D. A. Huffman, A method for the construction of minimum-redundancy codes. Proc. IRE, 40 (Sept. 1952) 1098-1101.

[19] P. Jacquet, and W. Szpankowski, Entropy computations via analytic depoissonization, Tech. Report CSD-TR-96-085, Purdue University (1996)

[20] P. Jacquet, and W. Szpankowski, Analytical depoissonization and its applications, Theoretical Computer Science, 201 (1-2) (1998) 1-62.

[21] D. Knuth, The Art of Computer Programming. Sorting and Searching. Vol. 3 (AddisonWesley, Reading MA, 1973).

[22] R. E. Krichevsky, "The Connection Between Redundancy and Reliability of Information about the Source," Probl. Inform. Trans., vol. 4, no. 3, pp. 48-57, 1968 (in Russian).

[23] R. E. Krichevsky, Optimal Source Coding Based on Observation, Probl. Inform. Trans., 11 (1) (1975) 37-48 (in Russian).

[24] R. E. Krichevsky and V. K. Trofimov, The Performance of Universal Encoding, IEEE Trans. Information Theory, 27 (1981) 199-207.

[25] R. E. Krichevsky, Universal Data Compression and Retrieval. (Kluwer, Norwell, MA, 1993). 
[26] R. E. Krichevskiy, Laplace's Law of Succession and Universal Encoding, IEEE Trans. Information Theory, 44 (1998) 296-303.

[27] A. Moffat and A. Turpin, On the Implementation of Minimum-Redundancy Prefix Codes, IEEE Trans. Communications, 45 (10) (1997) 1200-1207.

[28] V. I. Mudrov, An algorithm for enumeration of combinations, Vyc. Math. and Math. Phys., 5 (4) (1965) 776-778 (in Russian).

[29] Y.A. Reznik and W. Szpankowski, Asymptotic average redundancy of adaptive block codes, in Proc. Int. Symp. Information Theory (ISIT'03) (Yokohama, Japan, 29 June-4 July 2003) 79-.

[30] Y. A. Reznik, On second-order properties of minimum redundancy block codes, in Proc. Int. Symp. Information Theory (ISIT'04) (Chicago, IL, 27 June-2 July 2004) 94-.

[31] Y.A.Reznik, Asymptotic properties of sample-based entropy, information divergence, and related metrics, in Proc. Data Compression Conference (DCC'05) (Snowbird, UT, 29-31 March, 2005) 475-.

[32] Y.A.Reznik, Memory Efficient Encoding/Decoding of Variable-Length Codes for Monotonic Distributions, Data Compression Conference (DCC'07) - submitted.

[33] J. P. M. Schalkwijk, An Algorithm for Source Coding, IEEE Trans. Inform. Theory, 18 (3) (1972) 395-399.

[34] C. E. Shannon, A mathematical theory of communication, Bell Syst. Tech J. 27 (July 1948) 379-423.

[35] P. Stubley, On the redundancy of optimum fixed-to-variable length codes, Proc. Data Compression Conference (Snowbird, UT, 1994) 90-97.

[36] W. Szpankowski, Asymptotic Average Redundancy of Huffman (and Other) Block Codes, IEEE Trans. Information Theory, 46 (7) (2000) 2434-2443.

[37] W. Szpankowski, Average Case Analysis of Algorithms on Sequences, (John Wiley \& Sons, New York, 2001).

[38] T. Tjalkens, The complexity of minimum redundancy coding, in Proc. IEEE Int. Symp. Inf. Theory (ISIT'00), (Sorrento, Italy, 2000) 373-.

[39] T. Tjalkens, Implementation cost of the Huffman-Shannon-Fano code, in Proc. Data Compression Conference (DCC'05) (Snowbird, Utah, March 29-31, 2005) 123-132.

[40] Q. Xie and A. R. Barron, "Minimax redundancy for the class of memoryless sources," IEEE Trans. Inform. Theory, vol. 43, pp. 646-657, 1997.

[41] W. B. Pennebaker, J. L. Mitchell, G. G. Langdon, Jr., R. B. Arps An overview of the basic principles of the Q-Coder adaptive binary arithmetic coder IBM J. Res. Dev., 32 (6) (1988) 717-.

[42] ITU-T Recommendation T.82.

[43] D. Marpe, H. Schwartz, and T. Wiegand. Context-Based Adaptive Binary Arithmetic Coding in the H.264/AVC video compression standard. IEEE Trans. on CSVT, 13(7):620 636, July 2003.

[44] ISO/IEC 14496-10 (MPEG-4 AVC) / ITU-T Recommendation H.264 standard for video compression. 


\section{A Proof of Theorem 1}

We need to evaluate average redundancy of an adaptive code $\phi_{u}^{*}$ working with sam-

ples of length $t$ and blocks of length $n$ produced by a binary memoryless source with parameter $p$ :

$$
R_{\phi_{u}^{*}}(n, t, p)=\frac{1}{n} \sum_{|u|=t} \sum_{|w|=n} \operatorname{Pr}(u) \operatorname{Pr}(w)\left|\phi_{u}(w)\right|-H(p) .
$$

We will further assume that each codeword $\phi_{u}(w)$ is generated on the basis of KT-estimated probability $P_{K T}(w \mid u)$ (6), and therefore we can rewrite (20) as follows:

$$
R_{\phi_{u}^{*}}(n, t, p)=\frac{1}{n} \sum_{|u|=t} \sum_{|w|=n} \operatorname{Pr}(u) \operatorname{Pr}(w) \log P_{K T}^{-1}(w \mid u)-H(p)+\frac{1}{n} \Delta_{\phi_{u}^{*}}(n, t, p),
$$

where by

$$
\Delta_{\phi_{u}^{*}}(n, t, p)=\sum_{|u|=t} \sum_{|w|=n} \operatorname{Pr}(u) \operatorname{Pr}(w)\left[\left|\phi_{u}(w)\right|+\log P_{K T}(w \mid u)\right]
$$

we denote the redundancy of code $\phi_{u}^{*}$ with respect to the distribution it implements.

We know, that given density $P_{K T}(w \mid u)$ most existing minimum redundancy block codes (such as block Huffman, or Shannon algorithms) produce codewords such that:

$$
\left\lfloor\log P_{K T}(w \mid u)\right\rfloor \leqslant\left|\phi_{u}(w)\right| \leqslant\left\lceil\log P_{K T}(w \mid u)\right\rceil,
$$

which implies that $\Delta_{\phi_{u}^{*}}(n, t, p)$ is a quantity of bounded magnitude:

$$
\left|\Delta_{\phi_{u}^{*}}(n, t, p)\right| \leqslant 1,
$$

and which might have some erratic or oscillating behavior (cf. [36], [9]).

We now concentrate our attention on the main sum in (21):

$$
\begin{aligned}
& -\sum_{|u|=t} \sum_{|w|=n} \operatorname{Pr}(u) \operatorname{Pr}(w) \log P_{K T}(w \mid u)= \\
& \quad=-\sum_{|u w|=t+n} \operatorname{Pr}(u w) \log P_{K T}(u w)+\sum_{|u|=t} \operatorname{Pr}(u) \log P_{K T}(u) \\
& \quad=(t+n) C_{K T}(t+n, p)-t C_{K T}(t, p),
\end{aligned}
$$

where

$$
C_{K T}(n, p)=-\frac{1}{n} \sum_{|w|=n} \operatorname{Pr}(w) \log P_{K T}(w),
$$

is the average rate of the KT-estimator processing $n$-symbols words produced by $p$.

\section{A.1 Asymptotic average rates of empirical entropy and KT-estimator}

Consider KT-estimated probability of a word $w$

$$
P_{K T}(w)=\frac{\Gamma(k+1 / 2) \Gamma(n-k+1 / 2)}{\pi \Gamma(n+1)} .
$$


Using Stirling's approximation (and excluding cases when $k=0, n$ ), we can show that:

$$
\begin{aligned}
-\log P_{K T}(w)= & n F(w)+\frac{1}{2} \log n+\frac{1}{2} \log \frac{\pi}{2}+\frac{1}{12 n} \\
& +\frac{1}{24 k}+\frac{1}{24(n-k)}+O\left(\frac{1}{k^{3}}+\frac{1}{(n-k)^{3}}\right),
\end{aligned}
$$

where:

$$
F(w)=-\frac{k}{n} \log \left(\frac{k}{n}\right)-\frac{n-k}{n} \log \left(\frac{n-k}{n}\right) .
$$

is an empirical entropy [13, 24] of a word $w$.

The average rate of the empirical entropy $F(w)$ under source $p$ is:

$$
\begin{aligned}
& \sum_{|w|=n} \operatorname{Pr}(w) F(w) \\
& =-\sum_{k=0}^{n}\left(\begin{array}{l}
n \\
k
\end{array}\right) p^{k} q^{n-k}\left[\frac{k}{n} \log \left(\frac{k}{n}\right)+\frac{n-k}{n} \log \left(\frac{n-k}{n}\right)\right] \\
& =\log n-\sum_{k=1}^{n}\left(\begin{array}{l}
n \\
k
\end{array}\right) p^{k} q^{n-k} \frac{k}{n} \log (k)-\sum_{k=0}^{n-1}\left(\begin{array}{l}
n \\
k
\end{array}\right) p^{k} q^{n-k} \frac{n-k}{n} \log (n-k) \\
& =\log n-p \sum_{k=1}^{n}\left(\begin{array}{c}
n-1 \\
k-1
\end{array}\right) p^{k-1} q^{n-k} \log (k)-q \sum_{k=0}^{n-1}\left(\begin{array}{c}
n-1 \\
k
\end{array}\right) p^{k} q^{n-1-k} \log (n-k) \\
& =\log n-p f(n-1, k, p)-q f(n-1, k, q)
\end{aligned}
$$

where:

$$
f(n, k, \theta)=\sum_{k=0}^{n}\left(\begin{array}{l}
n \\
k
\end{array}\right) \theta^{k}(1-\theta)^{n-k} \log (1+k) .
$$

We immediately notice that (28) belongs to a class of so-called binomial sums (see, e.g. [3, p. 92]), and that for large $n$ we must have (a uniform in $\theta$ ) convergence $f(n, k, \theta) \rightarrow \log (1+\theta n)$. However, in order to obtain a more detailed asymptotic of $f(n, k, \theta)$, one must use analytic techniques, such as analytic depoissonization [19, 20], or singularity analysis of generating functions [15].

In fact, the last approach in application to a class of polylogarithmic Bernoulli sums has already been used by P. Flajolet [14, and in particular, he has shown that

$$
\sum_{k=1}^{n}\left(\begin{array}{l}
n \\
k
\end{array}\right) \theta^{k}(1-\theta)^{n-k} \log k=\log (\theta n)+\frac{\theta-1}{2 \theta n}-\frac{\theta^{2}-6 \theta+5}{12 \theta^{2} n^{2}}+O\left(\frac{1}{n^{3}}\right),
$$

which is a very similar sum to one that we need to evaluate (28). To take advantage of this existing result, we simply replace $\log (1+k)$ in (28) with:

$$
\log (k+1)=\log (k)+\frac{1}{k+1}+\frac{1}{2} \frac{1}{(k+1)(k+2)}+\frac{5}{6} \frac{1}{(k+1)(k+2)(k+3)}+\ldots
$$


The evaluation of the sums containing factorial powers of $k$ yields:

$$
\begin{aligned}
S_{1}(n, \theta) & =\sum_{k=1}^{n}\left(\begin{array}{l}
n \\
k
\end{array}\right) \theta^{k}(1-\theta)^{n-k} \frac{1}{k+1} \\
& =\frac{1}{\theta(n+1)}\left[1-(1-\theta)^{n}-\theta n(1-\theta)^{n}\right] \\
& =\frac{1}{\theta n}-\frac{1}{\theta n^{2}}+O\left(\frac{1}{n^{3}}\right) \\
S_{2}(n, \theta) & =\sum_{k=1}^{n}\left(\begin{array}{l}
n \\
k
\end{array}\right) \theta^{k}(1-\theta)^{n-k} \frac{1}{(k+1)(k+2)} \\
& =\frac{1}{\theta^{2}(n+1)(n+2)}\left[1-(1-\theta)^{n}-\theta n(1-\theta)^{n}-\frac{\theta^{2} n(n+1)}{2}(1-\theta)^{n}\right] \\
& =\frac{1}{\theta^{2} n^{2}}+O\left(\frac{1}{n^{3}}\right),
\end{aligned}
$$

and it is clear that the contribution of the subsequent terms in (29) to the sum (28) is within $O\left(\frac{1}{n^{3}}\right)$.

Combining the above formulae, we obtain

$$
f(n, k, \theta)=\log (\theta n)+\frac{1+\theta}{2 \theta n}-\frac{\theta^{2}+6 \theta-1}{12 \theta^{2} n^{2}}+O\left(\frac{1}{n^{3}}\right)
$$

and subsequently (after plugging (32) in (27) and some simple algebra):

$$
\sum_{|w|=n} \operatorname{Pr}(w) F(w)=H(p)-\frac{1}{2 n}+\frac{p q-1}{12 p q n^{2}}+O\left(\frac{1}{n^{3}}\right),
$$

which is an (up to $O\left(\frac{1}{n^{3}}\right)$-term) accurate asymptotic expression for the average rate of empirical entropy.

Remark 1 The average rate of empirical entropy has already been studied by Krichevsky (cf. [24, Lemma 1] and [25, Lemma 3.2.1]). His conclusion was that:

$$
-\frac{1}{n} \leqslant \sum_{|w|=n} \operatorname{Pr}(w) F(w)-H(p) \leqslant 0 .
$$

Our formula (33) confirms and refines this statement.

We now focus our attention on the average rate of the KT-estimator (24). Using our asymptotic expression (25) and replacing $\frac{1}{k}$ and $\frac{1}{n-k}$ with the appropriate factorial powers we can show that:

$$
\begin{aligned}
& C_{K T}(n, p)=-\frac{1}{n} \sum_{|w|=n} \operatorname{Pr}(w) \log P_{K T}(w) \\
&=\sum_{|w|=n} \operatorname{Pr}(w) F(w)+\frac{1}{n}\left\{\frac{1}{2} \log n+\frac{1}{2} \log \frac{\pi}{2}+\frac{1}{12 n}\right. \\
&\left.\quad+\frac{1}{24}\left[S_{1}(n, p)+S_{2}(n, p)\right]+\frac{1}{24}\left[S_{1}(n, q)+S_{2}(n, q)\right]\right\} \\
&+O\left(\frac{1}{n^{4}}\right),
\end{aligned}
$$


where $S_{1}(n, \theta)$ and $S_{2}(n, \theta)$ are already familiar sums (30) and (31).

Now by using (33) and expanding all the expressions in (34) we finally obtain:

$$
\begin{aligned}
C_{K T}(n, p)= & H(p)+\frac{1}{2 n}\left\{\log n+\log \frac{\pi}{2}-1-\frac{1-4 p q}{12 p q n}+\frac{1-3 p q}{12 p^{2} q^{2} n^{2}}\right\} \\
& +O\left(\frac{1}{n^{4}}\right) .
\end{aligned}
$$

\section{A.2 Asymptotic average rate of the adaptive block code}

Using (21,24) we can now say that

$$
R_{\phi_{u}^{*}}(n, t, p)=\frac{1}{n}\left[(t+n) C_{K T}(t+n, p)-t C_{K T}(t, p)-n H(p)+\Delta_{\phi_{u}^{*}}(n, t, p)\right],
$$

where $C_{K T}(n, p)$ is the average rate of the KT-estimator (24).

By applying our asymptotic result (35) for $C_{K T}(n, p)$ and combining the remaining (after some cancellations) terms we arrive at

$$
\begin{array}{r}
R_{\phi_{u}^{*}}(n, t, p)=\frac{1}{n}\left\{\frac{1}{2} \log \frac{t+n}{t}+\Delta_{\phi_{u}^{*}}(n, t, p)+\frac{1-4 p q}{24 p q} \frac{n}{t(t+n)}\right. \\
\left.-\frac{1-3 p q}{24 p^{2} q^{2}} \frac{(n+2 t) n}{t^{2}(t+n)^{2}}+O\left(\frac{1}{t^{3}}+\frac{1}{n^{3}}\right)\right\},
\end{array}
$$

which is formula (8) claimed by our theorem.

\section{B Example implementation of adaptive block coder}

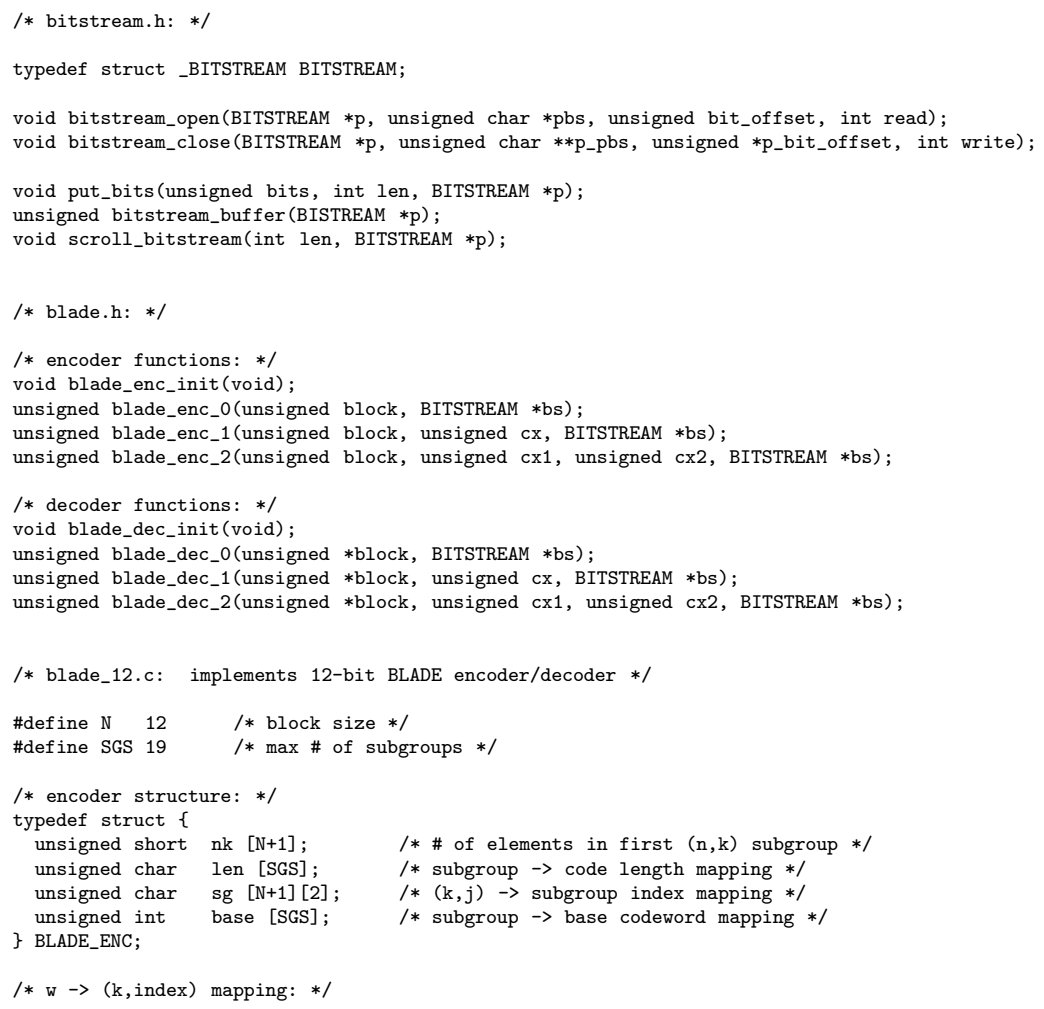




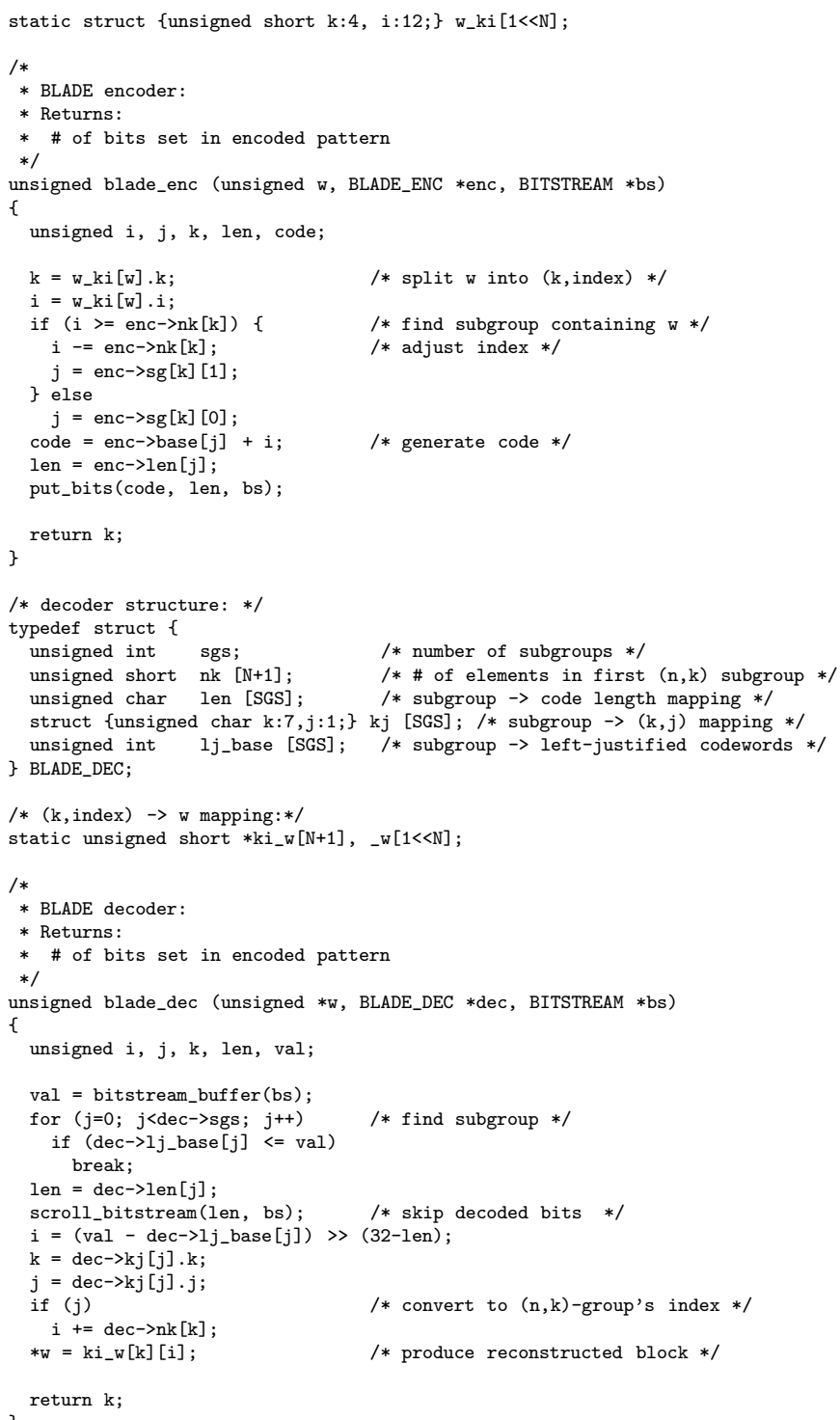


$\{1,12,66,220,495,303,924,792,495,219,66,4,1\},\{5,7,9,10,12,12,12,12,13,13,13,13,13,13,14,14\}$,

$\{\{0,0\},\{1,0\},\{2,0\},\{3,0\},\{4,0\},\{5,0\},\{11,0\},\{12,0\},\{5,1\},\{11,1\},\{6,0\},\{7,0\},\{9,0\},\{10,0\},\{9,1\},\{8,0\}\}$ $\{0 \times F 8000000,0 \times E 0000000,0 \times B F 000000,0 \times 88000000,0 \times 69100000,0 \times 56200000,0 \times 55 E 00000,0 \times 55 D 00000$, 0x46880000, 0x46480000, 0x29680000, 0x10A80000, 0x09D00000, 0x07C00000, 0x07BC0000, 0x000000000\} \}, $\{/ *(12,5): * / 15$

$\{1,12,66,220,495,792,509,792,350,220,66,12,1\},\{6,8,10,10,11,11,12,12,12,12,12,12,13,13,13\}$ $\{\{0,0\},\{1,0\},\{2,0\},\{12,0\},\{3,0\},\{11,0\},\{4,0\},\{5,0\},\{6,0\},\{8,0\},\{9,0\},\{10,0\},\{6,1\},\{8,1\},\{7,0\}\}$,

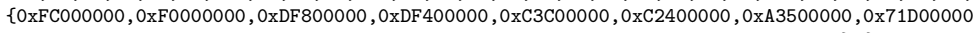
$0 \times 52000000,0 \times 3 \mathrm{C} 200000,0 \times 2 \mathrm{E} 600000,0 \times 2 \mathrm{~A} 400000,0 \times 1 \mathrm{D} 480000,0 \times 18 \mathrm{C} 00000,0 \times 00000000\}$

$\{/ *(12,6): * / 15$

$\{1,12,66,47,495,792,924,792,495,85,66,12,1\},\{8,8,9,9,11,11,11,11,12,12,12,12,12,12,13\}$,

$\{\{0,0\},\{12,0\},\{1,0\},\{11,0\},\{2,0\},\{3,0\},\{9,0\},\{10,0\},\{3,1\},\{9,1\},\{4,0\},\{5,0\},\{7,0\},\{8,0\},\{6,0\}\}$, \{0xFF000000, 0xFE000000, 0xF8000000, 0xF2000000, 0xE9C00000, 0xE3E00000, 0xD9400000, 0xD1000000, 0xC6300000, 0xBDC00000, 0x9ED00000, 0x6D500000, 0x3BD00000, 0x1CE00000, 0x00000000\} \},

$\{/ *(24,0): * / 19$,

$\{1,12,25,220,487,791,924,787,494,220,66,11,1\},\{1,5,9,10,13,16,17,19,20,22,24,25,26,27,28,30,31,32,32\}$,

$\{\{0,0\},\{1,0\},\{2,0\},\{2,1\},\{3,0\},\{4,0\},\{4,1\},\{5,0\},\{5,1\},\{6,0\},\{7,0\},\{7,1\},\{8,0\},\{8,1\},\{9,0\},\{10,0\},\{11,0\},\{11,1\},\{12,0\}\}$ $\{0 \times 80000000,0 \times 20000000,0 \times 13800000,0 \times 09400000,0 \times 02600000,0 \times 00790000,0 \times 00750000,0 \times 00122000,0 \times 00121000$,

Ox0003A000, 0x00008D00, 0x00008A80, 0x00000F00, 0x00000EE0, 0x00000120,0x000000018,0x00000002,0x00000001,0x00000000\} \},

$\{/ *(24,1): * / 17$,

$\{1,7,66,220,495,326,924,792,495,4,66,11,1\},\{1,5,6,9,12,15,17,18,20,22,23,24,25,26,27,28,28\}$,

$\{\{0,0\},\{1,0\},\{1,1\},\{2,0\},\{3,0\},\{4,0\},\{5,0\},\{5,1\},\{6,0\},\{7,0\},\{8,0\},\{9,0\},\{9,1\},\{10,0\},\{11,0\},\{11,1\},\{12,0\}\}$, $\{0 \times 80000000,0 \times 48000000,0 \times 34000000,0 \times 13000000,0 \times 05400000,0 \times 01620000,0 \times 00 B F 0000,0 \times 004$ A8000,

$0 \times 0010 \mathrm{C} 000,0 \times 00046000,0 \times 00008200,0 \times 00007 \mathrm{E} 00,0 \times 00001200,0 \times 00000180,0 \times 00000020,0 \times 000000010,0 \times 00000000\}\}$,

$\{/ *(24,2): * / 17$

$\{1,12,47,220,495,792,924,1,495,220,58,11,1\},\{2,5,8,9,11,14,16,18,19,20,21,22,23,24,24,25,25\}$,

$\{\{0,0\},\{1,0\},\{2,0\},\{2,1\},\{3,0\},\{4,0\},\{5,0\},\{6,0\},\{7,0\},\{7,1\},\{8,0\},\{9,0\},\{10,0\},\{10,1\},\{11,0\},\{11,1\},\{12,0\}\}$, $\{0 \times 6000000,0 \times 60000000,0 \times 31000000,0 \times 27800000,0 \times 0 C 000000,0 \times 04440000,0 \times 012 \mathrm{C} 0000,0 \times 00450000$,

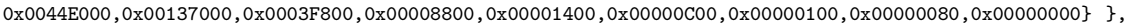

$\{/ *(24,3): * / 17$

$\{1,6,66,1,495,4,924,792,495,220,66,7,1\},\{2,5,6,8,10,11,13,14,15,16,18,19,20,21,21,22,22\}$,

$\{\{0,0\},\{1,0\},\{1,1\},\{2,0\},\{3,0\},\{3,1\},\{4,0\},\{5,0\},\{5,1\},\{6,0\},\{7,0\},\{8,0\},\{9,0\},\{10,0\},\{11,0\},\{11,1\},\{12,0\}\}$

$\{0 \times \mathrm{C} 0000000,0 \times 90000000,0 \times 78000000,0 \times 36000000,0 \times 35 \mathrm{C} 00000,0 \times 1 \mathrm{~A} 600000,0 \times 0 \mathrm{AE} 80000,0 \times 0 \mathrm{AD} 80000$,

0x04B00000,0x01140000,0x004E0000,0x00102000,0x00026000,0x00005000,0x00001800,0x00000400,0x000000000\} \},

$\{/ *(24,4): * / 15$

$\{1,12,66,220,495,10,924,792,495,220,66,7,1\},\{3,6,8,10,12,13,14,15,16,17,18,19,19,20,20\}$

$\{\{0,0\},\{1,0\},\{2,0\},\{3,0\},\{4,0\},\{5,0\},\{5,1\},\{6,0\},\{7,0\},\{8,0\},\{9,0\},\{10,0\},\{11,0\},\{11,1\},\{12,0\}\}$,

$\{0 \times \mathrm{xE} 0000000,0 \times \mathrm{BB} 0000000,0 \times 6 \mathrm{E} 000000,0 \times 37000000,0 \times 18100000,0 \times 17 \mathrm{C} 00000,0 \times 0 \mathrm{~B} 880000,0 \times 04500000$

$0 \times 01380000,0 \times 00408000,0 \times 00098000,0 \times 00014000,0 \times 00006000,0 \times 00001000,0 \times 00000000\}\}$,

$\{/ *(24,5): * / 16$

$\{1,12,66,220,495,792,451,792,2,220,66,11,1\},\{4,6,8,10,12,13,14,15,16,16,17,17,18,18,19,19\}$

$\{\{0,0\},\{1,0\},\{2,0\},\{3,0\},\{4,0\},\{5,0\},\{6,0\},\{6,1\},\{7,0\},\{8,0\},\{8,1\},\{9,0\},\{10,0\},\{11,0\},\{11,1\},\{12,0\}\}$,

$\{0 \times \mathrm{F} 0000000,0 \times \mathrm{C} 0000000,0 \times 7 \mathrm{E} 000000,0 \times 47000000,0 \times 28100000,0 \times 0 F 500000,0 \times 08440000,0 \times 04920000$

0x017A0000, 0x01780000, 0x00818000, 0x00138000, 0x00030000, 0x00004000, 0x000002000, 0x00000000\} \},

$\{/ *(24,6): * / 17$,

$\{1,8,65,220,2,792,924,792,495,220,59,12,1\},\{4,6,7,8,9,10,11,12,13,14,15,16,16,16,17,17,17\}$,

$\{\{0,0\},\{1,0\},\{1,1\},\{2,0\},\{2,1\},\{3,0\},\{4,0\},\{4,1\},\{5,0\},\{6,0\},\{7,0\},\{8,0\},\{9,0\},\{10,0\},\{10,1\},\{11,0\},\{12,0\}\}$, $\{0 \times \mathrm{xF} 0000000,0 \times \mathrm{x} 0000000,0 \times \mathrm{xC} 8000000,0 \times 87000000,0 \times 86800000,0 \times 4 \mathrm{~F} 800000,0 \times 4 \mathrm{~F} 400000,0 \times 30700000$,

0x17B00000, 0x09400000, 0x03100000, 0x01210000, 0x00450000, 0x000A0000, 0x00068000, 0x000008000, 0x00000000\} \},

$\{/ *(24,7): * / 15$

$\{1,12,66,220,495,62,924,792,495,220,66,8,1\},\{5,7,9,10,11,12,13,13,14,15,15,15,15,15,16\}$,

$\{\{0,0\},\{1,0\},\{2,0\},\{3,0\},\{4,0\},\{5,0\},\{5,1\},\{6,0\},\{7,0\},\{8,0\},\{9,0\},\{10,0\},\{11,0\},\{12,0\},\{11,1\}\}$,

$\{0 \times F 8000000,0 \times E 0000000,0 \times B F 000000,0 \times 88000000,0 \times 4 A 200000,0 \times 46400000,0 \times 2 F 700000,0 \times 12900000$,

$0 \times 06300000,0 \times 02520000,0 \times 009 \mathrm{~A} 0000,0 \times 00160000,0 \times 00060000,0 \times 00040000,0 \times 00000000\}\}$,

$\{/ *(24,8): * / 15$,

$\{1,12,66,220,287,792,924,792,495,220,62,12,1\},\{6,8,9,10,11,12,12,13,14,14,14,14,14,14,15\}$,

$\{\{0,0\},\{1,0\},\{2,0\},\{3,0\},\{4,0\},\{4,1\},\{5,0\},\{6,0\},\{7,0\},\{8,0\},\{9,0\},\{10,0\},\{11,0\},\{12,0\},\{10,1\}\}$,

$\{0 \times F C 000000,0 \times F 0000000,0 \times C F 000000,0 \times 98000000,0 \times 74200000,0 \times 67200000,0 \times 35 A 00000,0 \times 18 C 00000$,

$0 \times 0 \mathrm{C} 600000,0 \times 04 \mathrm{~A} 40000,0 \times 01340000,0 \times 003 \mathrm{C} 0000,0 \times 000 \mathrm{C} 0000,0 \times 00080000,0 \times 00000000\}$

$\{/ *(24,9): * / 14$

$\{1,12,66,220,417,792,924,792,495,220,66,12,1\},\{7,8,9,11,11,12,12,13,13,13,13,13,13,14\}$,

$\{\{0,0\},\{1,0\},\{2,0\},\{3,0\},\{4,0\},\{4,1\},\{5,0\},\{6,0\},\{7,0\},\{8,0\},\{10,0\},\{11,0\},\{12,0\},\{9,0\}\}$,

$\{0 \times F E 000000,0 \times F 2000000,0 x D 1000000,0 \times B 5800000,0 \times 81600000,0 \times 7 C 800000,0 \times 4 B 000000,0 \times 2 E 200000$,

$0 \times 15600000,0 \times 05 \mathrm{E} 80000,0 \times 03 \mathrm{D} 80000,0 \times 03780000,0 \times 03700000,0 \times 00000000\}\}$,

$\{/ *(24,10): * / 15$,

$\{1,12,66,220,221,792,923,792,495,220,66,12,1\},\{7,9,10,11,11,12,12,12,12,12,12,13,13,13,13\}$

$\{\{0,0\},\{1,0\},\{2,0\},\{3,0\},\{4,0\},\{4,1\},\{5,0\},\{6,0\},\{10,0\},\{11,0\},\{12,0\},\{6,1\},\{7,0\},\{8,0\},\{9,0\}\}$,

$\{0 \times F E 000000,0 \times F 8000000,0 \times E 7800000,0 \times C C 000000,0 \times B 0600000,0 \times 9 F 400000,0 \times 6$ DC00000, 0x34100000,

Ox2FF00000, 0x2F300000, 0x2F200000, 0x2F180000, 0x16580000, 0x06E00000, 0x00000000 \} \},

$\{/ *(24,11): * / 14$,

$\{1,12,23,220,495,792,924,792,495,220,66,12,1\},\{8,10,10,11,11,11,11,12,12,12,12,12,12,13\}$,

$\{\{0,0\},\{1,0\},\{2,0\},\{2,1\},\{3,0\},\{11,0\},\{12,0\},\{4,0\},\{5,0\},\{6,0\},\{8,0\},\{9,0\},\{10,0\},\{7,0\}\}$,

$\{0 x F F 000000,0 x F C 000000,0 x F 6400000,0 x F 0 E 00000,0 x D 5600000,0 x D 3 E 00000,0 x D 3 C 00000,0 x B 4$ D00000,

$0 \times 83500000,0 \times 49900000,0 \times 2 \mathrm{AA} 00000,0 \times 1 \mathrm{CE} 00000,0 \times 18 \mathrm{C} 00000,0 \times 00000000\}$

$\{/ *(24,12): * / 14$,

$\{1,12,66,220,495,792,504,792,495,220,66,12,1\},\{10,10,10,10,11,11,12,12,12,12,12,12,12,13\}$,

$\{\{0,0\},\{1,0\},\{11,0\},\{12,0\},\{2,0\},\{10,0\},\{3,0\},\{4,0\},\{5,0\},\{6,0\},\{7,0\},\{8,0\},\{9,0\},\{6,1\}\}$,

$\{0 \times F F C 00000,0 \times F C C 00000,0 \times F 9 C 00000,0 x F 9800000,0 \times F 1400000,0 \times E 9000000,0 \times D B 400000,0 \times B C 500000$,

\};

0x8AD00000, 0x6B500000, 0x39D00000, 0x1AE000000, 0x0D200000, 0x00000000\} \}

/* encoder tables (computed using decoder's tables): */

static BLADE_ENC enc_t $[1+(\mathrm{N} / 2+1)+(\mathrm{N}+1)]$;

/* initialize encoder: */

void blade_enc_init()

\{

unsigned int $i[\mathrm{~N}+1], j, k, 1$, w;

$/ *$ init enc []$: * /$

for $(j=0 ; j<1+(N / 2+1)+(N+1) ; j++)\{$

for $(k=0 ; k<=N ; k++)$ enc_t $[j] . n k[k]=d_{-1 e c} t[j] . n k[k]$

for $(k=0 ; k<=S G S ; k++)\{$ 


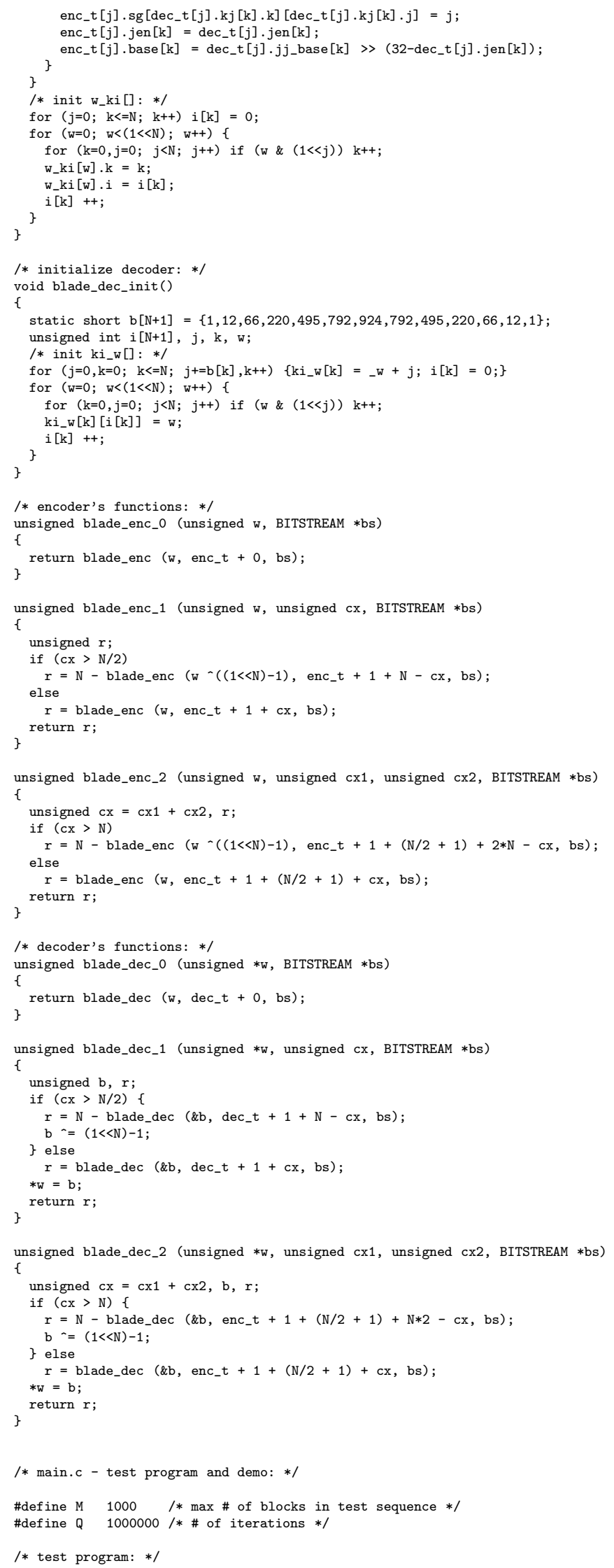




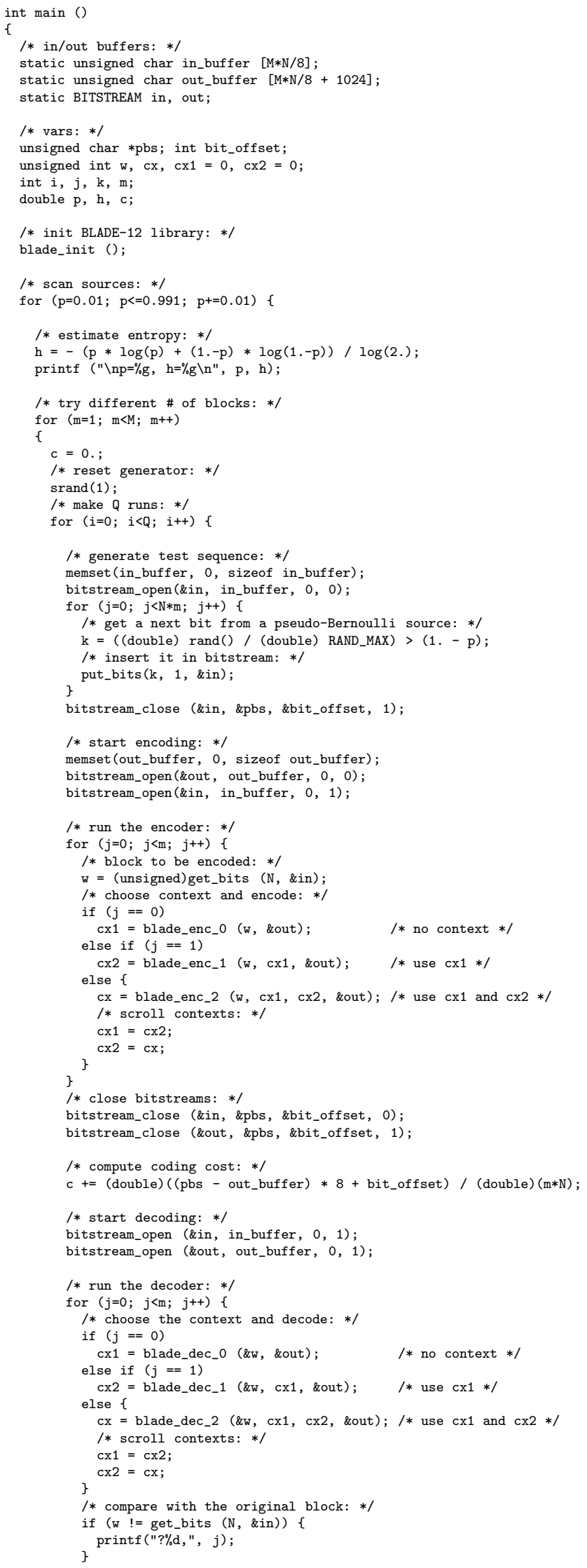


\}

/* close bitstreams: */

bitstream_close (\&in, \&pbs, \&bit_offset, 0);

bitstream_close (\&out, \&pbs, \&bit_offset, 0)

\}

/* print results: */

c $/=($ double $) Q$;

printf (" [\%d, \%g]

fflush (stdout)

\}

return 1;

\} 